\title{
APPLICATION OF RULED SURFACES IN FREEFORM AND GEAR METROLOGY
}

\author{
IVANa LinKeOVÁ ${ }^{a, *}$, Vít ZeLENÝ ${ }^{b}$ \\ ${ }^{a}$ Czech Technical University in Prague, Faculty of Mechanical Engineering, Department of Technical \\ Mathematics, Karlovo nám. 13, 12135 Prague, Czech Republic \\ ${ }^{b}$ Czech Metrology Institute, Laboratories of Fundamental Metrology, V Botanice 1504/4, 150 72 Prague, Czech \\ Republic \\ * corresponding author: Ivana.Linkeova@fs. cvut.cz
}

\begin{abstract}
An application of two ruled surfaces (i.e., surfaces generated by a motion of a straight line), a surface of hyperbolic paraboloid and a tangent surface of a cylindrical helix in freeform and gear metrology is introduced in this paper. Both surfaces have been implemented as the main functional figures in several artefacts - metrological calibration standards intended for testing the freeform capabilities of various measuring technologies (e.g., tactile point-to-point measurement and tactile scanning on coordinate measuring machine, optical scanning, computer tomography). Geometrical and mathematical properties of the surface used are summarised, CAD models of all the developed standards are presented and photos of the manufactured standards are shown.
\end{abstract}

KEYwords: Hyperbolic paraboloid, tangent surface of a helix, CAD model, freeform metrology, calibration standard.

\section{INTRODUCTION}

In precision engineering, an inspection of components with functional freeform surfaces lay great demands on metrological procedures. A freeform shape means a shape that does not belong to the basic geometrical elements (such as, plane, sphere, cylinder, cone, torus,...) and is difficult to describe it mathematically. Usually, the nominal shape and dimensions of the component with freeform surface is represented by CAD (Computer Aided Design) model and metrology procedure applied in inspection is a so called CADbased measurement. A reliable evaluation of freeform CAD-based measurement leads to the development of reference standards with a sufficient precision and specific properties. Calibration standards of regular shapes (basic geometrical elements) are well developed 1, while the traceability and quality inspection in freeform manufacturing are issues, due to the lack of traceable verification standards [2].

Based on a geometrical-mathematical approach, several freeform reference standards and gear measurement standard for testing the freeform and gear measurement capability of coordinate measuring machines and machine tools has been developed in the Czech Metrology Institute (CMI) in cooperation with the Czech Technical University in Prague (CTU) [3. The development of all the standards consists in a purposeful application of a surface of hyperbolic paraboloid and tangent surface of the cylindrical helix and effective usage of their excellent geometrical-mathematical properties [4].

Three of the standards developed, i.e. Freeform standard Hyperbolic paraboloid, Spatial hyperbolic paraboloid standard and Smart gear measurement standard, are mentioned in this paper. The paper is organised as follows. A mathematical model and geometrical properties of a surface of hyperbolic paraboloid and its application in the development of the Freeform standard Hyperbolic paraboloid is described in chapter 2 The solid of hyperbolic paraboloid applied in the Spatial hyperbolic paraboloid standard is presented in chapter 3 The relationship among the surfaces generated by screw motion of a circle involute, tangent surface of the cylindrical helix and flank surface of the involute gear is introduced in chapter 4 . Smart gear measurement standard that has been developed based on this relationship is mentioned in this chapter, too. For each standard, the mathematical model and geometrical properties of the main functional figure is described first. After that, the CAD model of the standard is introduced and finally, the photo of the manufactured standard is presented.

\section{Surface of HyPERBoliC PARABOLOID}

Consider a one-dimensional situation first. Given two points in three-dimensional space with Cartesian coordinate system $(O, x, y, z)$

$$
\mathbf{P}_{0}=\left(x_{0}, y_{0}, z_{0}\right), \mathbf{P}_{1}=\left(x_{1}, y_{1}, z_{1}\right)
$$

the straight line segment obtained by linear interpolation between these points is given by

$$
\mathbf{C}(u)=N_{0,1}(u) \mathbf{P}_{0}+N_{1,1}(u) \mathbf{P}_{1}, u \in[0,1]
$$


where $u$ is a parameter, $\mathbf{C}(u)$ is one-variate vector function and

$$
N_{0,1}(u)=1-u, N_{1,1}(u)=u
$$

are uniform B-spline basis functions of the first degree [5]. Equation (1) represents the straight line segment as a uniform clamped B-spline curve defined on a normalised domain of parameterization $u \in[0,1]$.

A surface of hyperbolic paraboloid is a linear interpolation among four points in the three-dimensional space. Denoting

$$
\begin{array}{ll}
\mathbf{P}_{00}=\left(x_{00}, y_{00}, z_{00}\right), & \mathbf{P}_{01}=\left(x_{01}, y_{01}, z_{01}\right), \\
\mathbf{P}_{10}=\left(x_{10}, y_{10}, z_{10}\right), & \mathbf{P}_{11}=\left(x_{11}, y_{11}, z_{11}\right),
\end{array}
$$

the surface of hyperbolic paraboloid (bilinear B-spline clamped surface defined on a normalised domain of parameterization $\left.(u, v) \in[0,1]^{2}\right)$ is given by

$$
\begin{aligned}
\mathbf{S}(u, v) & =(x(u, v), y(u, v), z(u, v))= \\
& =\left(\begin{array}{ll}
1-u & u
\end{array}\right) \cdot\left(\begin{array}{ll}
\mathbf{P}_{00} & \mathbf{P}_{01} \\
\mathbf{P}_{10} & \mathbf{P}_{11}
\end{array}\right) \cdot\left(\begin{array}{c}
1-v \\
v
\end{array}\right)
\end{aligned}
$$

with coordinate functions

$$
\begin{aligned}
x(u, v) & =(1-u)(1-v) x_{00}+(1-u) v x_{01}+ \\
& +u(1-v) x_{10}+u v x_{11} \\
y(u, v) & =(1-u)(1-v) y_{00}+(1-u) v y_{01}+ \\
& +u(1-v) y_{10}+u v y_{11} \\
z(u, v) & =(1-u)(1-v) z_{00}+(1-u) v z_{01}+ \\
& +u(1-v) z_{10}+u v z_{11}
\end{aligned}
$$

In computations, it is suitable to use an explicit expression of the surface of hyperbolic paraboloid. To be able to express the surface (3) explicitly, the orthogonal projection of quadrilateral $\mathbf{P}_{00} \mathbf{P}_{01} \mathbf{P}_{10} \mathbf{P}_{11}$ to the $(x, y)$ plane has to be an axis-aligned rectangle. Then, the explicit equation of the surface of hyperbolic paraboloid is given by

$$
z(x, y)=p+k(x-m)(y-n),
$$

where $\mathbf{V}=(m, n, p)$ is the vertex of the hyperbolic paraboloid and $k$ is a shape coefficient. Regarding the future application of this surface in the design of a freeform standard, consider the control vertices lying above the vertices of the axis-aligned square with centre at the origin of the coordinate system and length of the edge equal to $2 a$, fig. 1. Then

$$
\begin{array}{ll}
\mathbf{P}_{00}=\left(-a,-a, z_{00}\right), & \mathbf{P}_{01}=\left(-a, a, z_{01}\right), \\
\mathbf{P}_{10}=\left(a,-a, z_{10}\right), & \mathbf{P}_{11}=\left(a, a, z_{11}\right) .
\end{array}
$$

After substitution of control points coordinates (8) in (4) and (5), $x(u, v)$ and $y(u, v)$ coordinate functions are as follows

$$
x(u, v)=a(2 u-1), y(u, v)=a(2 v-1) .
$$

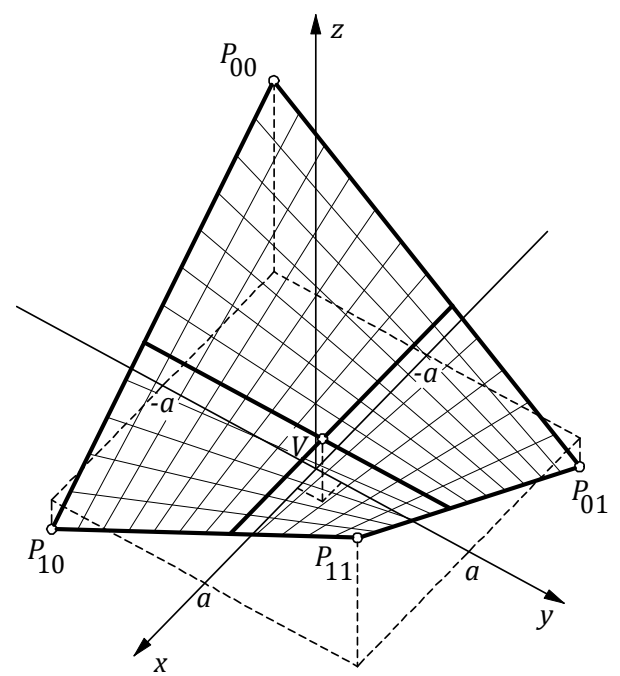

Figure 1. Surface of hyperbolic paraboloid.

Now, it is possible to solve (9) with respect to parameters $u$ and $v$

$$
u=\frac{x+a}{2 a}, v=\frac{y+a}{2 a}
$$

and substitute (10) in (6)

$$
\begin{aligned}
z(x, y) & =\frac{x y}{4 a^{2}}\left(z_{00}-z_{01}-z_{10}+z_{11}\right)+ \\
& +\frac{x}{4 a}\left(-z_{00}-z_{01}+z_{10}+z_{11}\right)+ \\
& +\frac{y}{4 a}\left(-z_{00}+z_{01}-z_{10}+z_{11}\right)+ \\
& +\frac{1}{4}\left(z_{00}+z_{01}+z_{10}+z_{11}\right) .
\end{aligned}
$$

Denote

$$
\begin{aligned}
& A=z_{00}-z_{01}-z_{10}+z_{11}, \\
& B=-z_{00}-z_{01}+z_{10}+z_{11}, \\
& C=-z_{00}+z_{01}-z_{10}+z_{11}, \\
& D=z_{00}+z_{01}+z_{10}+z_{11} .
\end{aligned}
$$

Then

$z(x, y)=\frac{1}{4}\left(D-\frac{C B}{A}\right)+\frac{A}{4 a^{2}}\left(x+\frac{C a}{A}\right)\left(y+\frac{B a}{A}\right)$.

Cartesian coordinates of the vertex of the hyperbolic paraboloid are

$$
m=-\frac{C a}{A}, n=-\frac{B a}{A}, p=\frac{1}{4}\left(D-\frac{C B}{A}\right)
$$

and shape coefficient

$$
k=\frac{A}{4 a^{2}} .
$$

If the centre of the square, above whose vertices the control points lie, is shifted to point $(q, r, 0)$, the coordinates of the hyperbolic paraboloid vertex are

$$
m=q-\frac{C a}{A}, n=r-\frac{B a}{A}, p=\frac{1}{4}\left(D-\frac{C B}{A}\right) .
$$


Considering (7), a point on the surface is obtained by a substitution of a pair of variables $x=\alpha$ and $y=\beta,(\alpha, \beta) \in R^{2}$. Specially, the control points (8) are the following function values of surface 7

$$
\begin{aligned}
& \mathbf{P}_{00}=z(-a,-a), \mathbf{P}_{01}=z(-a, a), \\
& \mathbf{P}_{10}=z(a,-a), \mathbf{P}_{11}=z(a, a)
\end{aligned}
$$

and vertex

$$
\mathbf{V}=z(m, n) .
$$

There are three types of curves on the surface of hyperbolic paraboloid: two systems of parametric straight lines, two systems of parabolas and one system of hyperbolas. The parametric straight lines (by means of which the surface in fig. 1 is depicted) are obtained by a substitution of the constant value $x=\alpha$ or $y=\beta$ in 7

$$
\begin{aligned}
& z(x, \beta)=k(\beta-n) x+p-k m(\beta-n), \\
& z(\alpha, y)=k(\alpha-m) y+p-k n(a-m) .
\end{aligned}
$$

Equations (16) and (17) geometrically express the intersection of the surface of the hyperbolic paraboloid and planes parallel with $(x, z)$ and $(y, z)$ planes in the given order. Specially, the boundaries of the hyperbolic paraboloid are obtained by

$$
z(x,-a), z(x, a), z(-a, y), z(a, y)
$$

and the straight lines passing through the vertex (red lines on fig. 22 are given by

$$
z(x, n)=p, z(m, y)=p .
$$

Obviously, equations (16) and 17) represent two systems of straight lines on the surface of hyperbolic paraboloid. Each straight line from the first system intersects all straight lines from the second system and is perpendicular to them. Straight lines 18 passing through the vertex are parallel with $(x, y)$ plane.

Two system of parabolas on the surface of hyperbolic paraboloid are obtained as intersections of the surface and planes parallel with the plane $y=x$ or $y=-x$, i.e.

$$
\begin{aligned}
& z(x, x+d)= \\
& =k x^{2}-(m+d-n) k x+k m(n-d)+p, \\
& z(x,-x+d) \\
& =-k x^{2}+(m+d-n) k x+k m(n-d)+p,
\end{aligned}
$$

where $d$ is $y$-intercept of the plane $y=x+d$ or $y=-x+d$. Specially, the parabolas passing through the vertex are given by

$$
\begin{gathered}
z(x, x)=p+k(x-m)(x-n) \\
z(x,-x)=p+k(x-m)(-x-n) .
\end{gathered}
$$

Equation 19 represents the blue parabola and 20 the green parabola in fig. 2 .
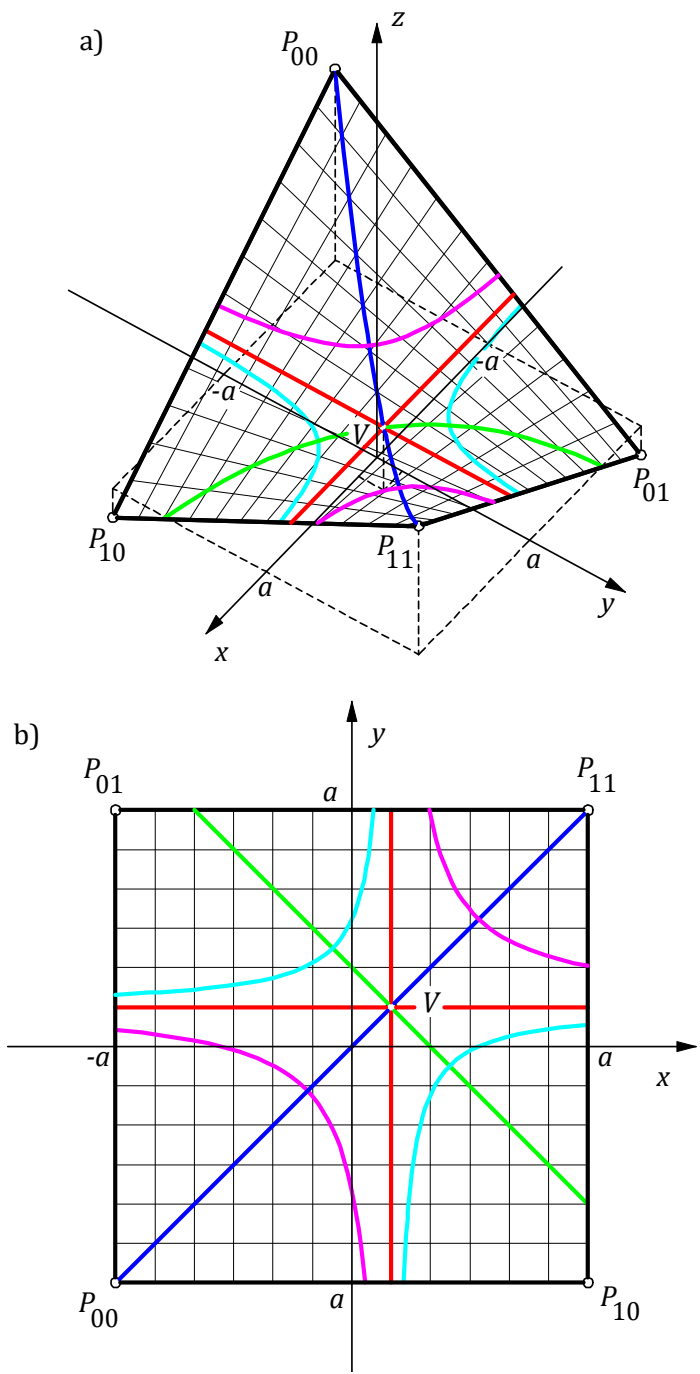

Figure 2. Curves on surface of hyperbolic paraboloid a) axonometric view, b) top view.

Hyperbolas on the surface of hyperbolic paraboloid are obtained as intersections of the surface and planes parallel with $(x, y)$ plane, i.e. $z=\gamma$, where $\gamma$ is a real constant

$$
p+k(x-m)(y-n)=\gamma .
$$

Specially, for $\gamma=p$ we get $(18)$, i.e. asymptotes of all the hyperbolas in the top view, see red straight lines in fig. 2 b). The magenta hyperbola in fig. 2 corresponds to $\gamma>p$, the cyan hyperbola corresponds to $\gamma<p$.

\subsection{FreEForm STANDARD HyPERBOLIC PARABOLOID}

The CAD model of the standard is depicted in fig. 3 a). The surface of hyperbolic paraboloid (green) is created in the CAD system as bilinear B-spline surface given by control points

$$
\begin{array}{ll}
\mathbf{P}_{00}=(-9,-9,73), & \mathbf{P}_{01}=(-9,87,-11) \\
\mathbf{P}_{10}=(87,-9,-11), & \mathbf{P}_{11}=(87,87,49)
\end{array}
$$


a)

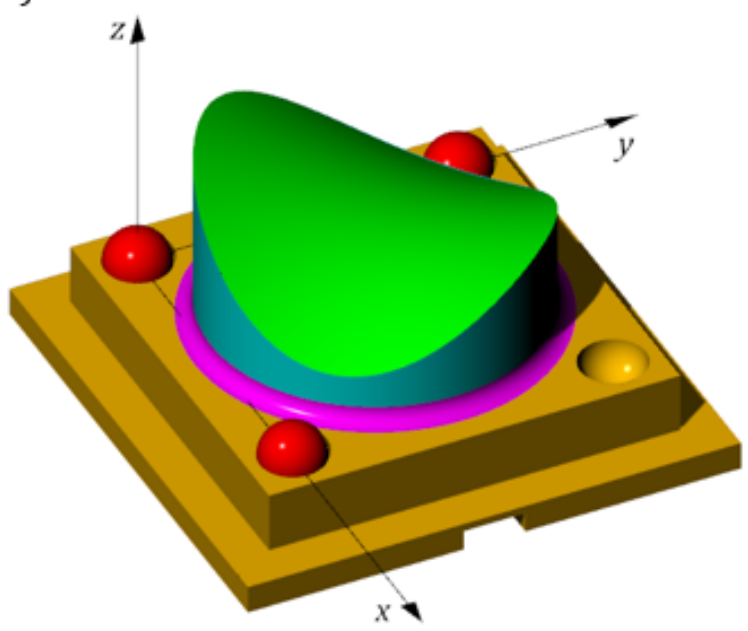

c)

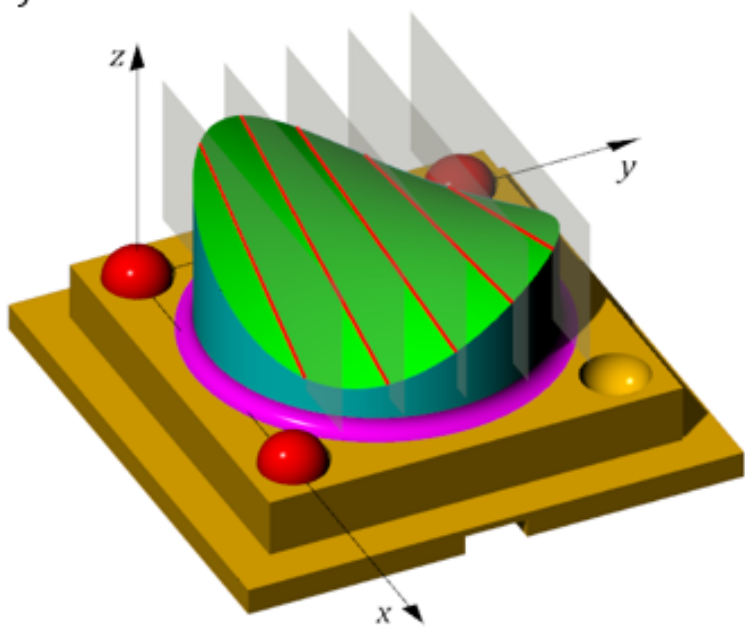

e)

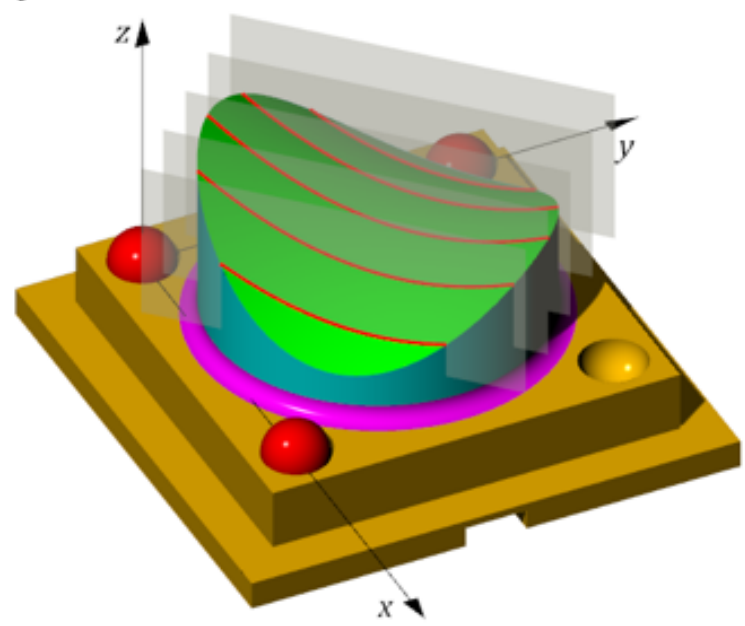

b)

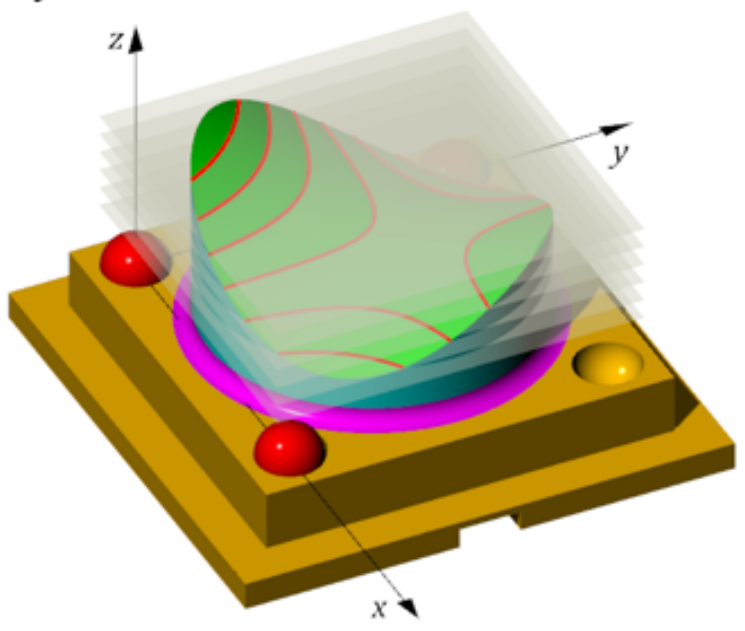

d)

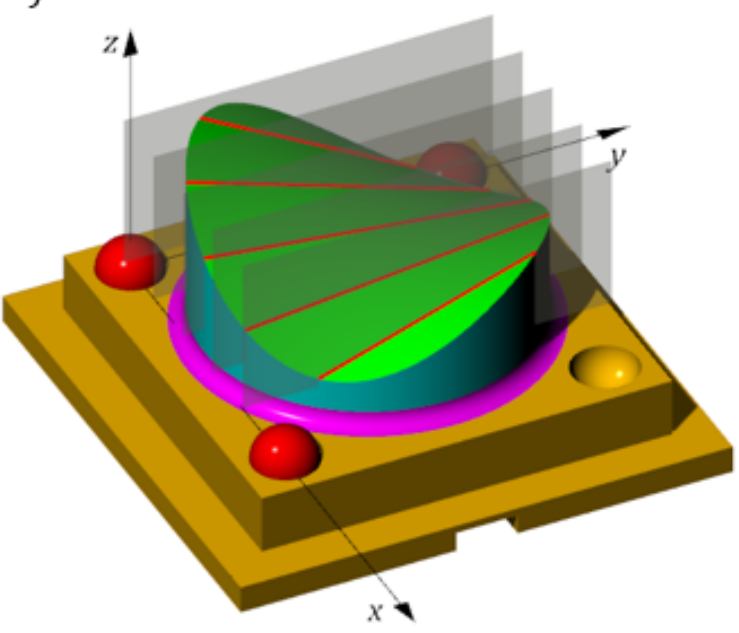

f)

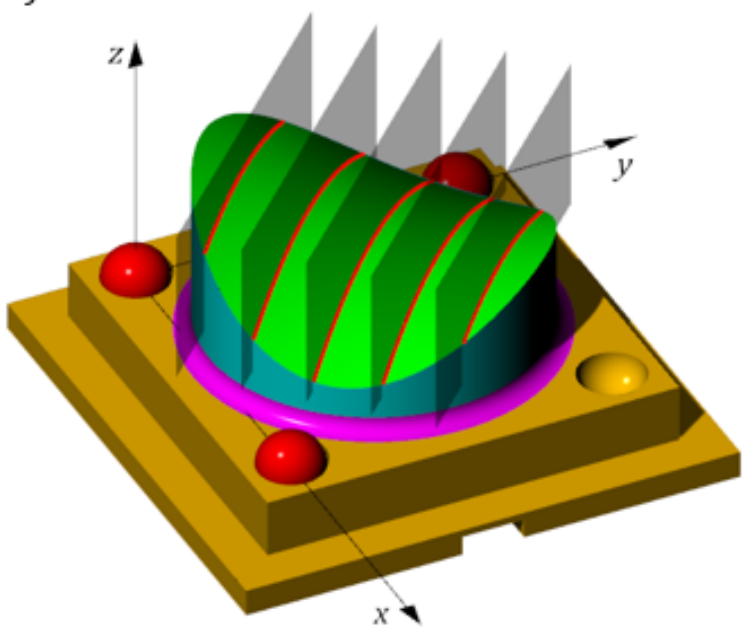

Figure 3. Freeform standard Hyperbolic paraboloid

a) CAD model, b) Hyperbolic sections by planes parallel with $(x, y)$ plane, c) Linear sections by planes parallel with $(x, z)$ plane, d) Linear sections by planes parallel with $(y, z)$ plane, e) Parabolic sections by planes parallel with plane $y=x, \mathrm{f})$ Parabolic sections by planes parallel with plane $y=-x$. 
Control points 22 are located above the vertices of axis aligned square with the centre at point $(q, r, 0)=(39,39,0)$ and length of the edge $2 a=96$ (all dimensions are in $\mathrm{mm}$ ). Using (12), 14) and 15 . the explicit equation of the functional surface of the standard is given by

$$
z(x, y)=24+\frac{1}{64}(x-47)(y-47) .
$$

This equation is useful when evaluating the measured data for both the tactile point-to-point measurement and the tactile scanning on coordinate a measurement machine.

The standard of dimensions $120 \times 120 \times 67 \mathrm{~mm}$ consists of a step-squared base intended for clamping the standard on the coordinate measuring machine (depicted in yellow in fig. 3). Three precise reference spheres (red) with a radius equal to $8 \mathrm{~mm}$ are glued into the three of the four spherical holes on the standard. These spheres serve for s workpiece coordinate system definition. The surface of hyperbolic paraboloid (green) is trimmed by the cylindrical surface (blue) with an axis parallel to $z$-axis and radius equal to $40 \mathrm{~mm}$. The common boundary between the upper squared face and the cylinder is filled with a radius of $4 \mathrm{~mm}$, i.e. the transition surface is created by a part of torus (magenta).

The surface of hyperbolic paraboloid used in the Freeform standard hyperbolic paraboloid allows to measure the following curves.

Hyperbolas. The hyperbolic sections 21 of the hyperbolic paraboloid by planes parallel with $(x, y)$ plane are depicted in fig. $3 \mathrm{~b}$ ).

Straight lines. The linear sections (16) and (17) of the hyperbolic paraboloid by planes parallel with $(x, z)$ and $(y, z)$ planes are depicted in fig. $3 \mathrm{c})$ and fig. 3 d) in the given order.

Parabolas. The parabolic sections 19$)$ and $(20)$ of the hyperbolic paraboloid by planes parallel with the plane $y=x$ and $y=-x$ are depicted in fig. 3 e) and fig. $3 \mathrm{f}$ ) in the given order.

\subsection{PhysicAl FREEFORM STANDARD HYPERBOLIC PARABOLOID}

The standard has been manufactured by 3 -axis milling on numerically controlled milling machine US20 by high speed cutting from steel EN X10CrNi18-9.The calibration of the standard on the coordinate measuring machine is shown in fig. 4 .

\section{SOlid OF HYPERBOLIC PARABOLOID}

The solid of the hyperbolic paraboloid represents an extension of linear interpolation among eight points in three-dimensional space. Considering the following

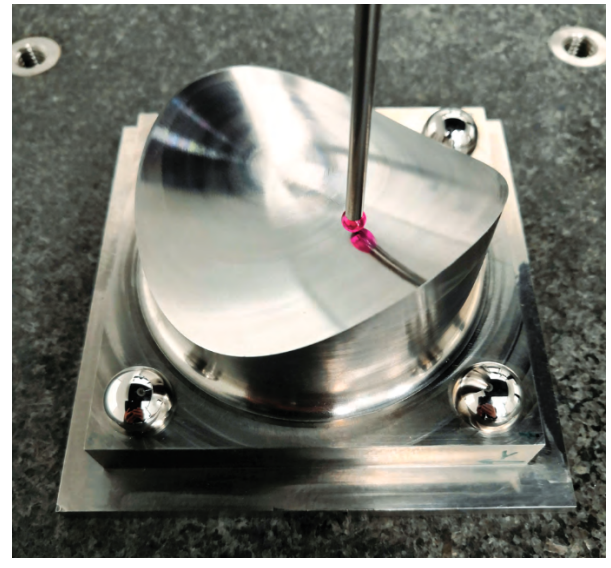

FiguRE 4. Calibration of freeform standard Hyperbolic paraboloid on coordinate measurement machine.

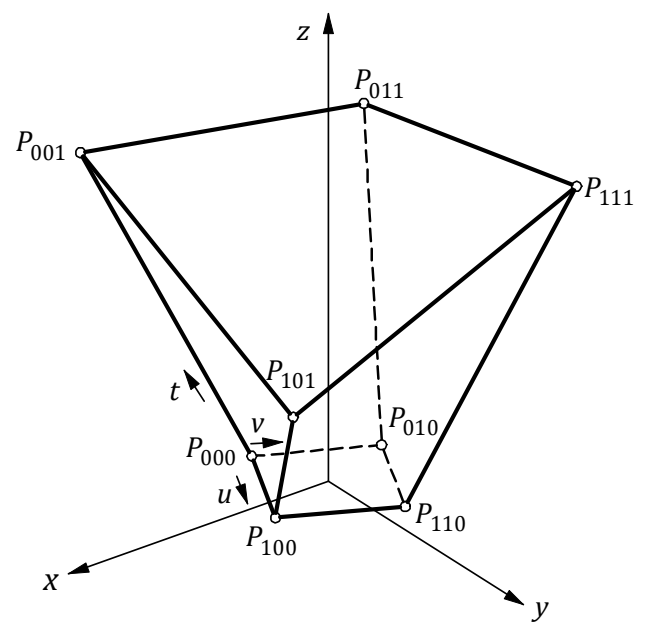

Figure 5. Solid of hyperbolic paraboloid.

control points

$$
\begin{aligned}
& \mathbf{P}_{000}=\left(x_{000}, y_{000}, z_{000}\right), \mathbf{P}_{010}=\left(x_{010}, y_{010}, z_{010}\right), \\
& \mathbf{P}_{100}=\left(x_{100}, y_{100}, z_{100}\right), \mathbf{P}_{110}=\left(x_{110}, y_{110}, z_{110}\right), \\
& \mathbf{P}_{001}=\left(x_{001}, y_{001}, z_{001}\right), \mathbf{P}_{011}=\left(x_{011}, y_{011}, z_{011}\right), \\
& \mathbf{P}_{101}=\left(x_{101}, y_{101}, z_{101}\right), \mathbf{P}_{111}=\left(x_{111}, y_{111}, z_{111}\right),
\end{aligned}
$$

the trilinear uniform clamped B-spline solid defined on a normalised domain of parameterization $(u, v, t) \in$ $[0,1]^{3}$ is given by

$$
\mathbf{B}(u, v, t)=\sum_{i=0}^{1} \sum_{j=0}^{1} \sum_{k=0}^{1} N_{i, 1}(u) N_{j, 1}(v) N_{k_{1}}(t) \mathbf{P}_{i j k},
$$

see example in fig. 5

If one of the parameters in 23 is constant, a parametric surface of the solid is obtained. For $u=\alpha$, $v=\beta$ and $t=\gamma, \alpha, \beta, \gamma \in[0,1]$, there are three systems of parametric surfaces

$$
\mathbf{B}(u, v, \gamma)=\sum_{i=0}^{1} \sum_{j=0}^{1} \sum_{k=0}^{1} N_{i, 1}(u) N_{j, 1}(v) N_{k, 1}(\gamma) \mathbf{P}_{i j k}
$$




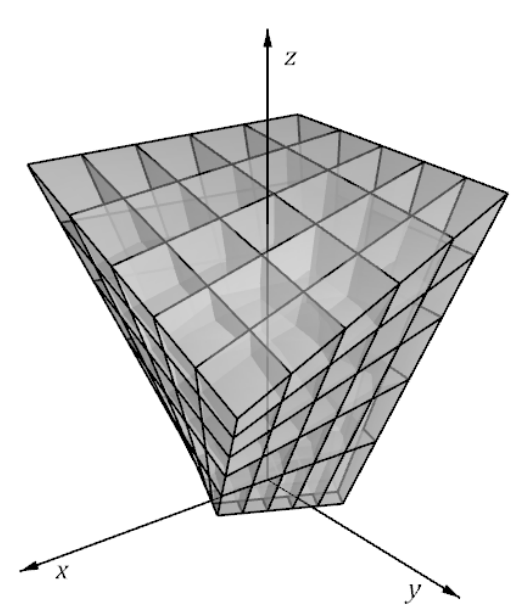

Figure 6. Parametric surfaces in solid of hyperbolic paraboloid.

$$
\begin{aligned}
\mathbf{B}(u, \beta, t) & =\sum_{i=0}^{1} \sum_{j=0}^{1} \sum_{k=0}^{1} N_{i, 1}(u) N_{j, 1}(\beta) N_{k, 1}(t) \mathbf{P}_{i j k}, \\
\mathbf{B}(\alpha, v, t) & =\sum_{i=0}^{1} \sum_{j=0}^{1} \sum_{k=0}^{1} N_{i, 1}(\alpha) N_{j, 1}(v) N_{k, 1}(t) \mathbf{P}_{i j k} .
\end{aligned}
$$

Obviously, all three systems of parametric surfaces of the solid are created by surfaces of hyperbolic paraboloid. Consequently, the parametric curves of the solid are straight lines (fig. 6).

\subsection{SPATIAL hyperbolic PARABOLOID STANDARD}

Spatial hyperbolic paraboloid standard with cavities has been developed so that the standard can be calibrated by the point-to-point tactile measurement and then measured by means of various metrology technologies (tactile scanning, optical scanning, computer tomography, etc.). The solid of hyperbolic paraboloid (23) represents the freeform functional figure on this standard, see fig. 7, where the CAD model of the standard is depicted. The standard of dimensions $100 \times 100 \times 100 \mathrm{~mm}$ consists of squared box base intended for clamping the standard to the coordinate measuring machine. The centre upper squared face of the base lies at the origin of the coordinate system. Freeform faces of the standard are the boundary surfaces of the solid of hyperbolic paraboloid. The cavity of the standard is created by non-coaxial cylindrical holes serving for orientation when measuring the standard by a computer tomography technology.

\subsubsection{Physical Spatial hyperbolic PARABOLOID STANDARD}

The standard has been manufactured from aluminium alloy CERTAL EN AW 7022 [AlZn5Mg3Cu] by numerically controlled machining and polished. The calibration of the standard on coordinate measuring machine is shown in fig. 8

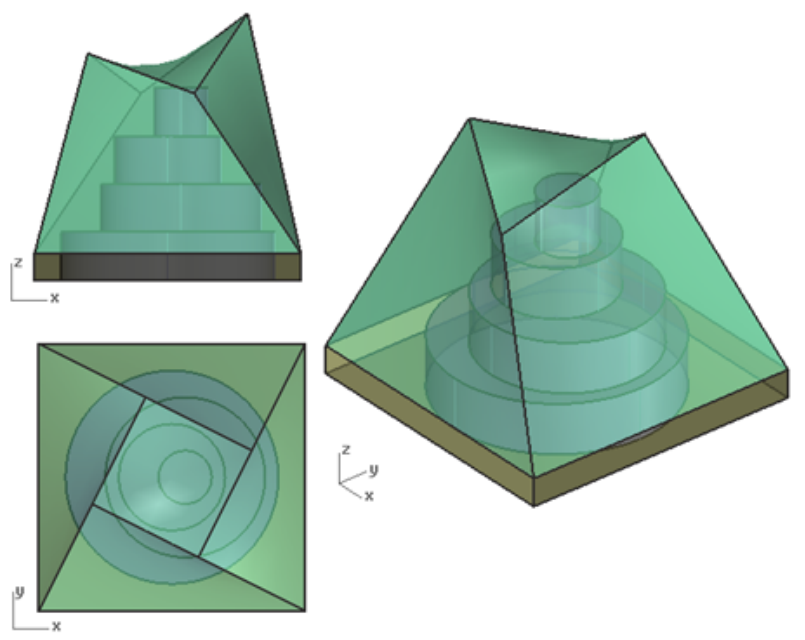

Figure 7. CAD model of Spatial hyperbolic paraboloid standar

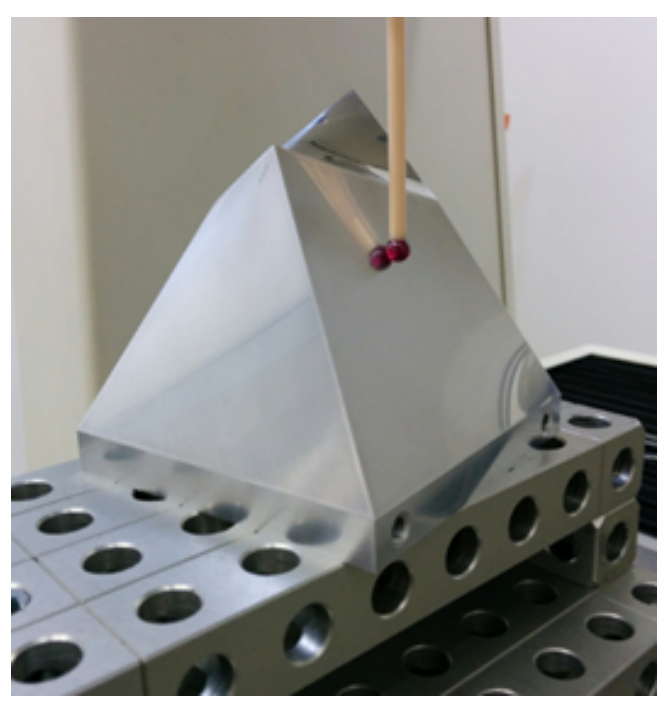

FiguRE 8. Calibration of Spatial hyperbolic paraboloid standard.

\section{Surfaces Generated By motion OF A CIRCLE INVOLUTE}

Involute $e$ of a curve $c$ is the trajectory of a free endpoint of the tangent line of the curve $c$ rolling along the curve $c$. In other words, the normal line at each point of the involute $e$ is the tangent line to the curve $c$.

In technical practice, a circle involute (hereinafter referred to as the "involute"), see fig. 9. has a special meaning. It is well known that a flank surface of involute spur gears is an involute ruled surface (i.e., the surface generated by a translation of the involute along a straight line) and a flank surface of involute helical gears is an involute helicoidal surface (i.e., the surface generated by screw motion of the involute). What is not so obvious is that the involute helicoidal surface and tangent surface of the helix have the same shape (if certain geometrical conditions are met). Consequently, the flank surface of involute helical gears 


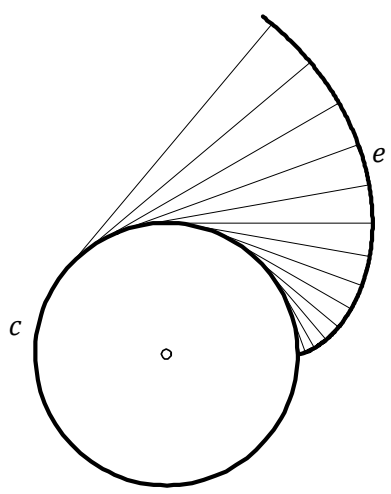

Figure 9. Circle involute.

can be expressed as a tangent surface of the base helix, which is mathematically simpler than an expression of an involute helicoidal surface [6].

In this section, the helix, as a trajectory of a point under screw motion, is described firstly. After that, the tangent surface of the helix, involute helicoidal surface and ruled surface are mentioned together with their properties. Finally, an example of an application of these surfaces in metrology practice is given.

\subsection{TAngent Surface of a CYlindrical HELIX}

The tangent surface of a cylindrical helix is very important in gear geometry because an unmodified involute helical tooth flank is a tangent surface of the base helix 7], i.e., the helix located on the gear base cylinder.

Helix is a spatial curve generated by screw motion of a generating point. A screw motion is a combination of revolution about axis $o$ of the screw motion and a translation along axis $o$. The helix is called cylindrical if the angle of revolution is directly proportional to the length of translation and the distance of the generating point from the axis $o$ is constant. The cylindrical helix (hereinafter referred to as the "helix") is a curve of a constant slope, i.e. the angle formed by the tangent line to the helix and any plane perpendicular to axis $o$ is constant. The development of the helix (flattening onto a plane) is a directly proportional graph with the revolution (measured in the length of arc) on horizontal axis and translation of vertical axis. The slope of the graph is identical to the slope of the helix. The tangent surface of the helix is a developable surface [8] (it is possible to unfold it into a plane without any distortion).

To derive a mathematical model of the tangent surface of the helix, consider the cylinder (axis identical to coordinate $z$ axis, radius $r_{b}$, height equal to the lead $p_{z}$ ) and one thread of right hand helix $h_{b}$ generated by a screw motion of point $\mathbf{B}$ located on coordinate $x$ axis,

$$
\mathbf{B}=\left(x_{\mathbf{B}}, y_{\mathbf{B}}, z_{\mathbf{B}}, 1\right)=\left(r_{b}, 0,0,1\right)
$$

(in homogeneous coordinates), see fig. 10

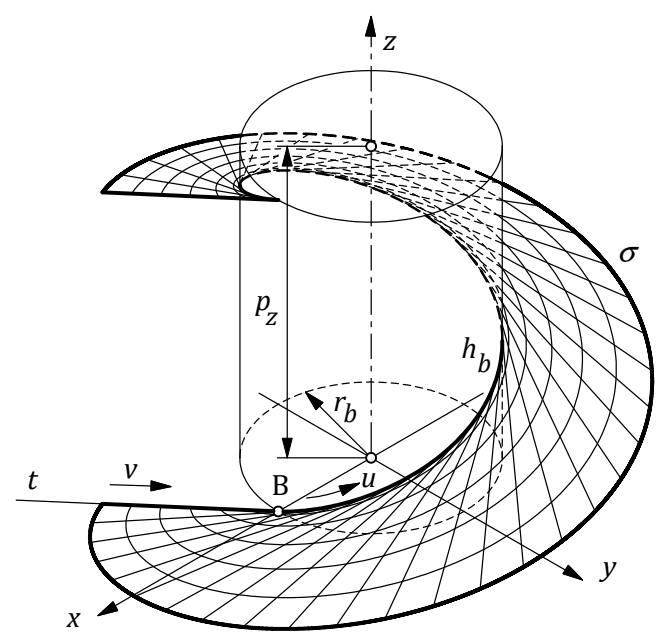

Figure 10. Tangent surface of cylindrical helix.

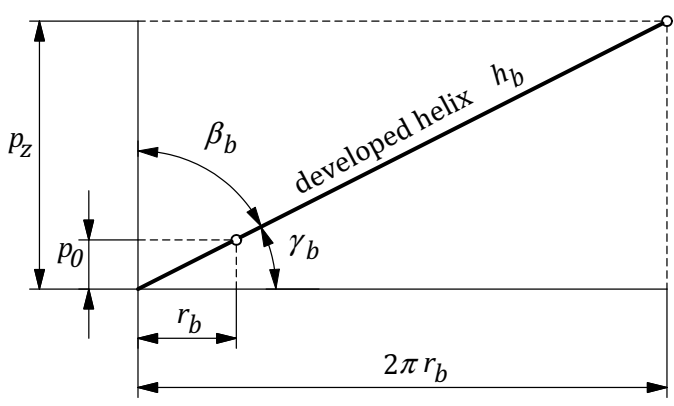

Figure 11. Development of the helix.

The development of the cylinder is the rectangle (width $2 \pi r_{b}$, height $p_{z}$ ), the development of the helix is the diagonal of this rectangle, see fig. 11. Direction vector $\mathbf{b}$ of tangent line $t$ is given by

$$
\mathbf{b}=\left(b_{1}, b_{2}, b_{3}, 0\right)=\left(0, r_{b}, p_{0}, 0\right)
$$

where $p_{0}=p_{z} / 2 \pi$ is called the parameter of the screw motion (translation directly proportional to the revolution about one radian).

The vector equation of tangent line

$$
t: \mathbf{T}(v)=(x(v), y(v), z(v), 1)
$$

to the helix $h_{b}$ passing through point $\mathbf{B}$ is given by

$$
\begin{aligned}
\mathbf{T}(v) & =\left(x_{\mathbf{B}}+b_{1} v, y_{\mathbf{B}}+b_{2} v, z_{\mathbf{B}}+b_{3} v, 1\right)= \\
& =\left(r_{b}, r_{b} v, p_{0} v, 1\right), v \in \mathbb{R} .
\end{aligned}
$$

The transformation matrix of the right hand screw motion is given by

$$
\mathbf{G}_{R}(u)=\left(\begin{array}{cccc}
\cos u & \sin u & 0 & 0 \\
-\sin u & \cos u & 0 & 0 \\
0 & 0 & 1 & 0 \\
0 & 0 & p_{0} u & 1
\end{array}\right), u \in \mathbb{R}
$$

where $u$ is the angle parameter in radians. The first three rows of matrix 25 correspond to the revolution 


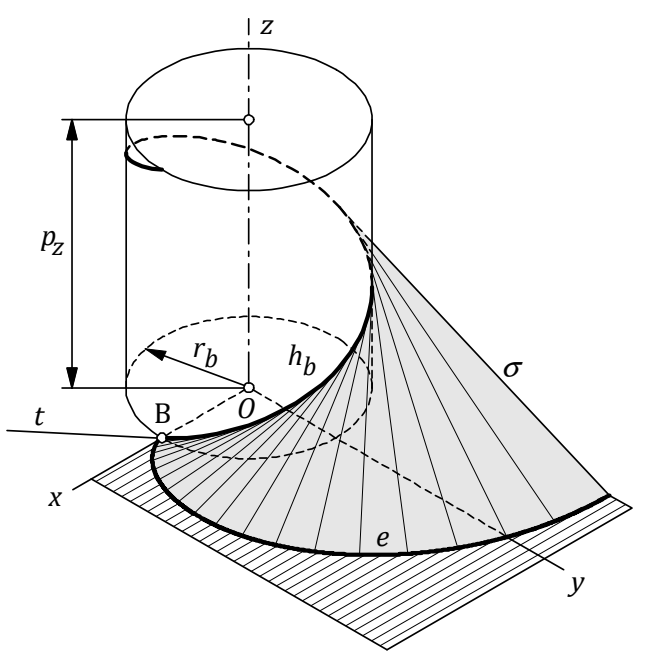

FiguRE 12. Involute section $e$ of tangent surface of the helix.

about $z$ axis and the fourth row corresponds to the translation along $z$ axis.

Tangent surface

$$
\sigma: \mathbf{S}(u, v)=(x(u, v), y(u, v), z(u, v), 1)
$$

of the right hand helix is given by

$$
\begin{aligned}
& \mathbf{S}(u, v)=\mathbf{T}(v) \cdot \mathbf{G}_{R}(u)= \\
= & \left(r_{b}(\cos u-v \sin u), r_{b}(\sin u+v \cos u), p_{0}(v+u), 1\right) .
\end{aligned}
$$

There are three types of curves on the surface $(26)$ : two systems of parametric curves, i.e. helices and straight lines, see fig. 10, and involute curves, see fig. 12. Two parametric curves, each of them from a different system, have a common point located on the surface, parametric coordinates of this point correspond to the constant values of parameters $u$ and $v$. Parametric $u$-curves are helices obtained by the substitution of constant value $v=v_{c}, v_{c} \in \mathbb{R}$ in 26 . Parametric $v$-curves are tangent lines to the helix obtained by the substitution of constant value $u=u_{c}$, $u_{c} \in \mathbb{R}$ in 26 . The involute curve is the intersection of the tangent surface of the helix and any plane perpendicular to $z$ axis.

To derive the equation of the involute curve from (26), the solution of equation $z(u, v)=z_{c}, z_{c} \in \mathbb{R}$ has to be substituted in $(26)$. Considering $z_{c}=0$, i.e., the intersection of the tangent surface of the helix and coordinate plane $(x, y)$, the condition $p_{0}(u+v)=0$ is met if $v=-u$ or $u=-v$. These solutions are identical from the geometrical point of view, because the shape of involute curves obtained by substituting $v=-u$ as well as $u=-v$ in $(26)$ is the same. However, from mathematical point of view, the later solution and the interchange of parameters $u$ and $v$ is preferable. Thus, the involute $e$ obtained by substituting $u=v$ in 26

$$
e: \mathbf{E}(v)=\left(x_{\mathbf{E}}(v), y_{\mathbf{E}}(v), z_{\mathbf{E}}(v), 1\right)
$$

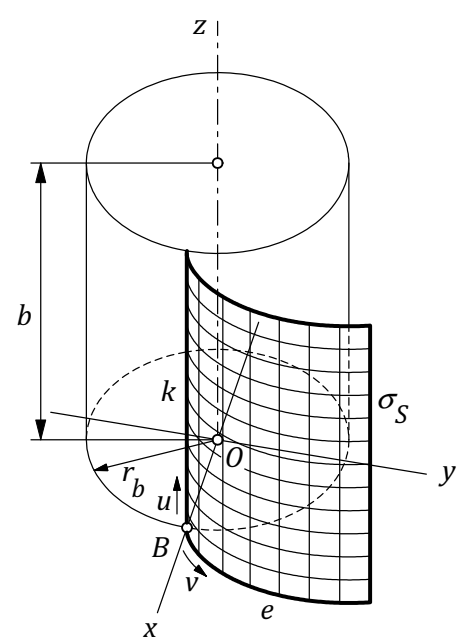

FigURE 13. Ruled involute surface.

is given by

$$
\begin{aligned}
\mathbf{E}(v)= & \left(r_{b}(\cos v+v \sin v), r_{b}(\sin v-v \cos v), 0,1\right) \\
& v \geq 0
\end{aligned}
$$

Note that the flank surface of a helical gear can be generated by a screw motion of the involute profile, too.

\subsection{InVOlute RULED SURFACE}

The surface of the involute spur tooth flank $\sigma_{S}$ : $\mathbf{S}_{S}(u, v)$ is generated by a translation of involute $e$ given by (27) along a straight line $k \| z$

$\mathbf{S}_{S}(u, v)=\left(r_{b}(\cos v+v \sin v), r_{b}(\sin v-v \cos v), u, 1\right)$,

see fig. 13 Parametric $u$-curves are straight lines parallel with $z$ axis, parametric $v$-curves are involutes (27) lying in planes perpendicular to $z$ axis.

\subsection{SMART GEAR MEASUREMENT STANDARD}

In gear metrology, precise gear measurement standards of various designs and purposes are developed, manufactured and calibrated. For example, for a profile and helix inspection, the Internal involute scanning measurement standards developed by PhysikalischTechnische Bundesanstalt (PTB) contains both the precise internal and external involute profiles [9] and profiles with a certain waviness superposed [10], which enable to characterise the dynamic behaviour of the probing system. The Large gear measurement standard (PTB) is designed as a segment of a complete gear [11] and large ring gear measurement standard (PTB) embodies three different internal and external gearing, each one with helix angles of $0^{\circ}$ (spur gearing), $10^{\circ}$ and $20^{\circ}$ [12. All the specially designed or commercially produced gear measurement standards for the profile and helix inspection contain separate geometry for each type of gearing. 


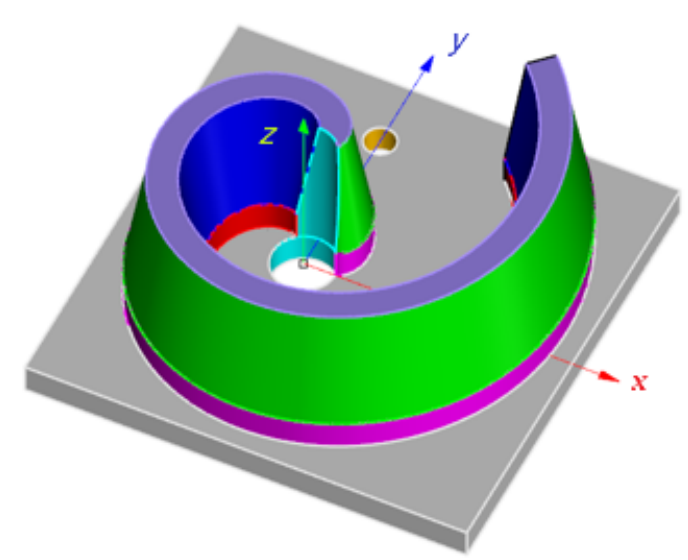

Figure 14. CAD model of Smart gear standard.

The contrary, the flank surface of the Smart gear measurement standard (developed by the CMI in cooperation with CTU) is represented by a tangent surface of the helix on which all three types of curves (straight lines, involutes and helices) important in gear profile and helix inspection are located. Consequently, it is possible to measure profile and helix deviations of different gearings on one geometry. The CAD model of the Smart gear measurement standard is shown in (fig. 14).

Note that both the helix and involute are transcendental curves, i.e., they have neither polynomial nor rational parameterisation. Consequently, it is impossible to express them by NURBS (Non-Uniform $\mathrm{B}$-Spline) representation, which is the theoretical basis of CAD systems. Thus, their precise CAD model cannot be created. Usually, more or less accurate approximation by means of B-spline curves is used in CAD systems [13. Involute is not implemented in CAD systems yet.

The standard has a box base $140 \times 140 \times 10 \mathrm{~mm}$ (drawn in grey in fig. 14). The origin of the coordinate system lying in the upper face of the box is determined by the centre of a cylindrical hole with the base diameter $d_{b}$ (cyan). The direction of $y$-axis is defined by the centre of the cylindrical hole diameter of $10 \mathrm{~mm}$ (yellow). The upper face of the box defines $(x, y)$ plane, $z$ axis is perpendicular to it. The $10 \mathrm{~mm}$ high involute ruled surface placed on the upper face of the box represents the external and internal spur involute flank surface (magenta and red). The external and internal helical involute flank surface (green and blue) is represented by the tangent surface of the base helix trimmed by planes $z=10$ and $z=50 \mathrm{~mm}$ (violet).

The most important shape figure included in the tangent surface of the base helix corresponds to the real helical gear with parameters given in tab. 11 This gear, together with its spur variant (with $\left(\beta=0^{\circ}\right)$ and the other parameters the same as is given in tab. 1), is shown in fig. 15 .

\begin{tabular}{lcc}
\hline Parameter & Symbol & Value \\
\hline Helix angle & $\beta\left(^{\circ}\right)$ & 10 \\
Normal module & $m_{n}(\mathrm{~mm})$ & 3.54 \\
Teeth number & $z(-)$ & 6 \\
Reference circle diameter & $d(\mathrm{~mm})$ & 21.324 \\
Tip circle diameter & $d_{a}(\mathrm{~mm})$ & 28.324 \\
Normal angle & $\alpha_{n}\left(^{\circ}\right)$ & 20 \\
Transverse angle & $\alpha_{t}\left(^{\circ}\right)$ & 20.284 \\
Base circle diameter & $d_{b}(\mathrm{~mm})$ & 20.002 \\
Lead & $p_{z}(\mathrm{~mm})$ & 379.926 \\
\hline
\end{tabular}

TABLE 1. Gear parameters of Smart gear measurement standard.

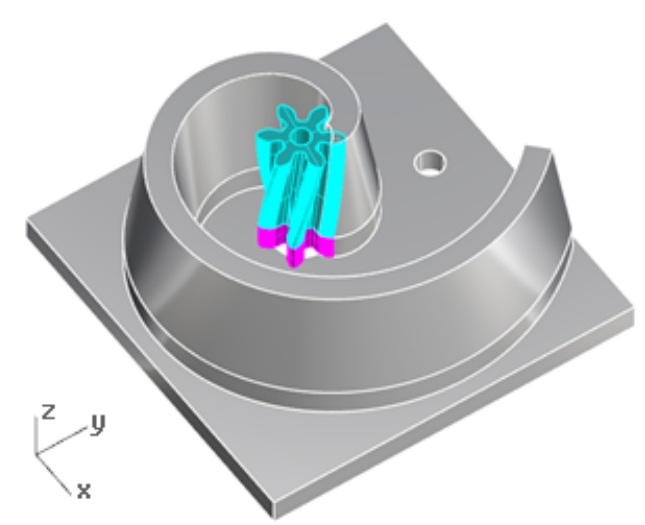

Figure 15. Spur (magenta) and helical (cyan) gear included in Smart gear measurement standard.

The tangent surface of the base helix used in the Smart gear standard allows to measure the following curves.

Helices Helices located on the tangent surface of the helix are defined as intersections of the surface and cylindrical surface coaxial with the base cylinder. In fig. 16 the intersection with the cylindrical surface of radius $r=34.911 \mathrm{~mm}$ and the corresponding angle $\beta=30^{\circ}$ can be seen. The shape of the standard enables to measure the external helical gear from $\beta=0^{\circ}$ (spur gear as a special case of the helical gear) to $\beta=60^{\circ}$ and internal helical gear from $\beta=0^{\circ}$ to $\beta=56^{\circ}$. Parameters of the selected external helical gear helices which possible to measure are given in tab. 2

The corresponding helical teeth included in the standard are depicted in fig. 17 together with their helices lying on the surface of the standard.

Straight lines Straight lines located on the tangent surface of the helix are defined by individual tangent lines of the helix. Consequently, these straight lines are obtained as intersections of the surface and tangent planes to the base cylinder, see fig. 18. Here, several section planes perpendicular to $(x, y)$ plane with angle span of $45^{\circ}$ depicted. 


\begin{tabular}{cccc}
\hline$\beta\left(^{\circ}\right)$ & $m_{n}(\mathbf{m m})$ & $z(-)$ & $d(\mathbf{m m})$ \\
\hline 10 & 3.5 & 6 & 21.324 \\
15 & 1.49 & 21 & 32.404 \\
20 & 2.76 & 15 & 44.016 \\
25 & 3.01 & 17 & 56.392 \\
30 & 2.02 & 30 & 69.821 \\
35 & 3.02 & 23 & 84.679 \\
40 & 5.98 & 13 & 101.476 \\
45 & 4.50 & 19 & 120.934 \\
\hline
\end{tabular}

TABLE 2. Parameters of selected helical gears on Smart gear standard

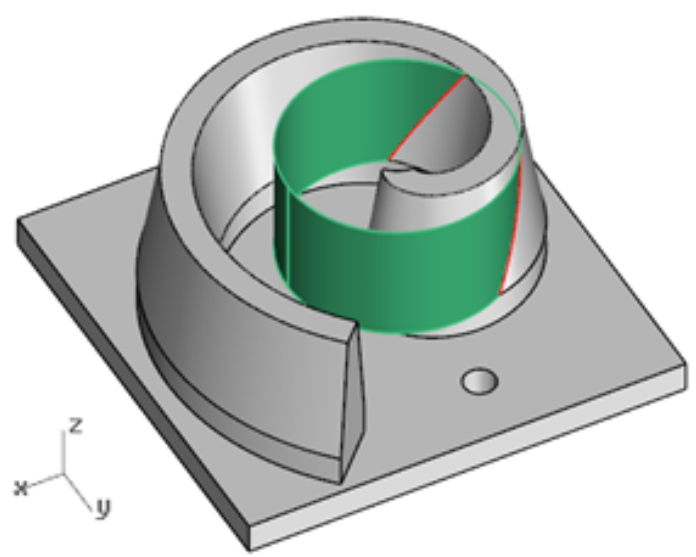

Figure 16. Helical sections on Smart gear measurement standard.

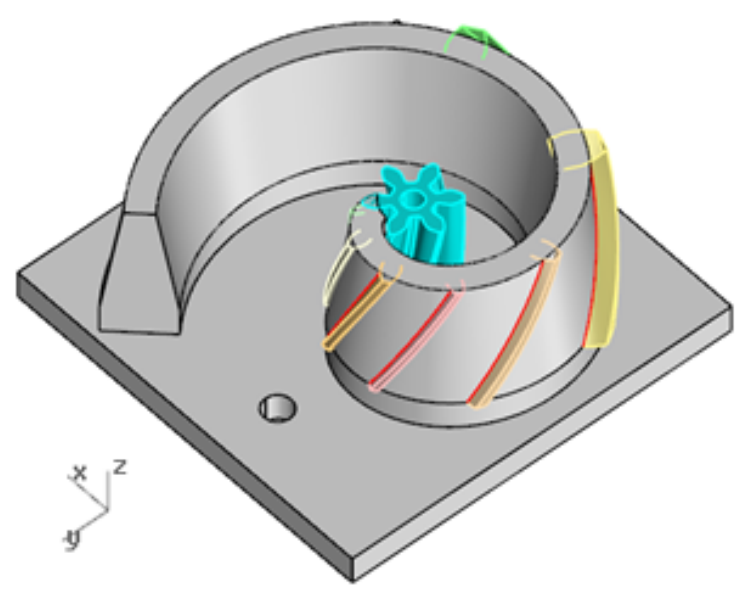

Figure 17. Helical teeth included in Smart gear measurement standard.

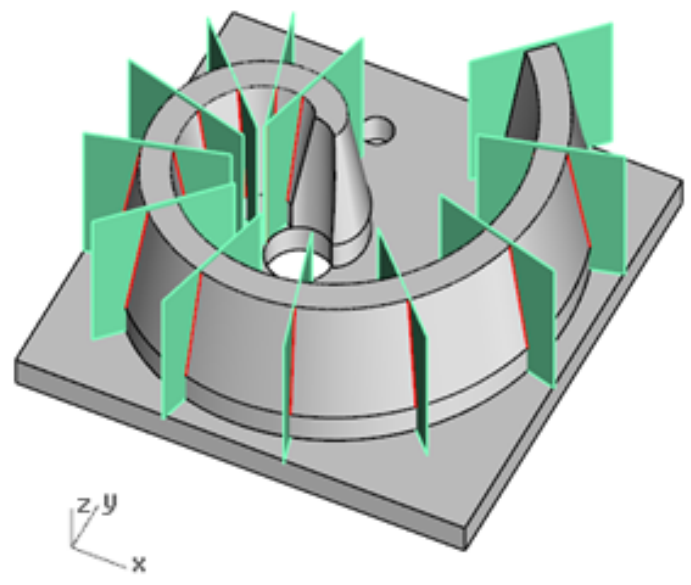

Figure 18. Line sections on Smart gear measurement standard.

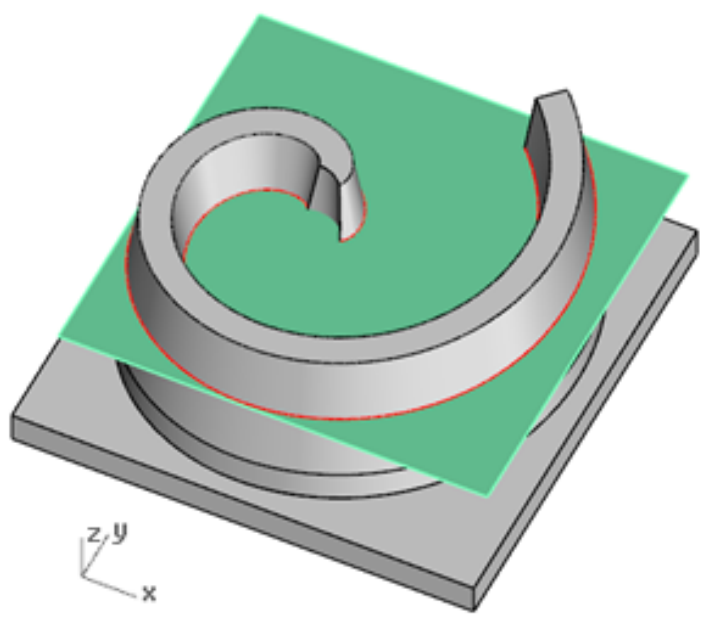

Figure 19. Involute sections on Smart gear measurement standard.

Involutes Involutes located on the tangent surface of the helix are defined as intersections of the surface and planes perpendicular to the axis of the helix. The plane $z=30 \mathrm{~mm}$ is depicted in fig. 19 .

\subsubsection{Physical Smart gear Standard}

The final shape of the Smart gear standard is currently in the research and development phase. Therefore, the first sample of this standard has been manufactured by $3 \mathrm{D}$ printing technology and experimental measurements have been realised. Based on the metrological experience, the shape of the standard will be modified. Numerically controlled manufacturing technology and metal material is assumed for the future production of the standard. Due to the geometrical properties of the flank surfaces, using the 5-axis flank milling technology to obtain as precise model as possible, the main functional surface of the standard will be possible. 


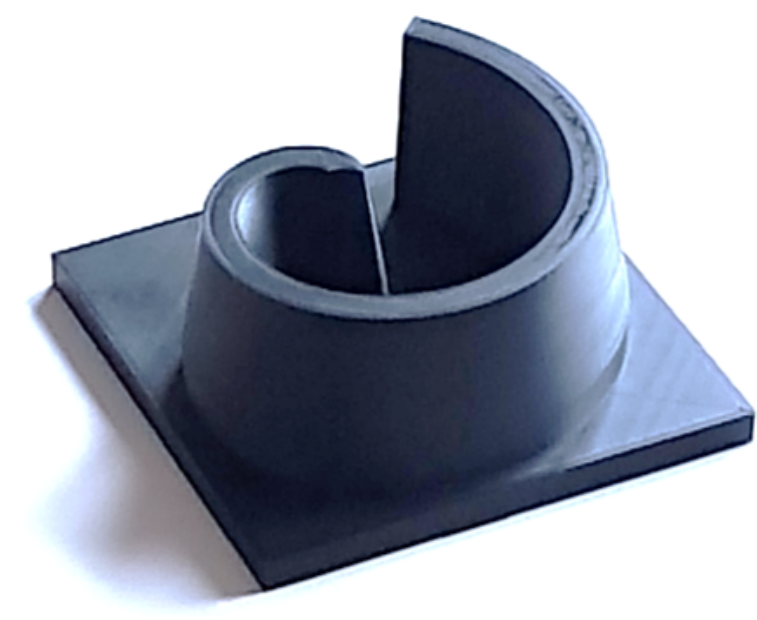

FiguRE 20. Smart gear measurement standard manufactured by $3 \mathrm{D}$ printing technology.

\section{Conclusion}

Two ruled surfaces - surface of hyperbolic paraboloid and tangent surface of a cylindrical helix are described in this paper. Geometrical-mathematical properties of both surfaces are summarised and their application in freeform and gear metrology is shown. Two freeform metrological standards - Freeform standard Hyperbolic paraboloid and Spatial hyperbolic paraboloid standard and Smart gear measurement standard developed by the CMI in cooperation with the CTU are introduced.

All of the above mentioned artefacts are considered to be the calibration standards for freeform metrology - a new, rapidly evolving specialisation focused on the quality inspection of generally shaped parts. The main objective of involving these artefacts in freeform metrology is to determine the best measuring capabilities of coordinate measuring machines working on different principles. Usually, the basic measurand in freeform metrology is a form deviation, i.e. the normal distance between the actual point located on the measured workpiece and nominal point located on the theoretical CAD model of the measured surface. The geometrical properties of all the above mentioned artefacts allow to use of advanced measurement procedures, such as measurement of straight lines and curves located on the freeform surface. Consequently, it is possible to measure surface roughness and waviness along the surface lines. All the artefacts have been applied not only in a determination of measurement uncertainty of tactile and optical measuring machines and in an inter-laboratory comparison at the national level by the CMI laboratories but also, in the case of the CMI freeform standard Hyperbolic paraboloid, at the international level.

\section{REFERENCES}

[1] B. Ačko, M. McCarthy, F. Haertig. Standards for testing freeform measurement capability of optical and tactile co-ordinate measuring machines. Meas Sci Technol 23(9):23094013, 2012.

[2] G. Roger, D. Flack, M. McCarthy. A review of industrial capabilities to measure freeform surfaces. NPL report, DEPCEM 0142007.

[3] V. Zelený, I. Linkeová, P. Skalník. Calibration of freeform standard. euspen's 15th International Conference 8 Exhibition, Leuven, Belgium 2015.

[4] I. Linkeová, P. Skalník, V. Zelený. Calibrated cad model of freeform standard. XXI IMEKO World Congress Measurement in Research and Industry, Prague 2015.

[5] L. Piegl, W. Tiller. The NURBS book. Springer-Verlag Berlin Heidelberg 1997. DOI:10.1007/978-3-642-59223-2.

[6] V. Zelený, I. Linkeová, J. Sýkora, P. Skalník. Mathematical approach to evaluate involute gear profile and helix deviations without using special gear software. Mechanism and Machine Theory 135:150 - 164, 2019. DOI:10.1016/j.mechmachtheory.2019.01.030.

[7] H. Stachel. On Spatial Involute Gearing. 6th International Conference on Applied Informatics (ICAI), Eger, Hungary (2004):27 - 39.

[8] H. Stachel. Comments on Helical Developables. Proceedings of the 13th International Conference on Geometry and Graphics, Dresden, Germany (2008).

[9] M. Stein. Internal involute scanning measurement standard. [2019-12-31], https://www.ptb.de/emrp/eng56-standards.html

[10] M. Stein. Internal involute wavines scanning measurement standard. [2019-12-31], https://www.ptb.de/emrp/eng56-standards.html

[11] M. Stein. Large gear measurement standard. [2019-12-31], https://www.ptb.de/emrp/eng56-standards.html

[12] M. Stein. Large ring gear measurement standard. [2019-12-31], https://www .ptb.de/emrp/eng56-standards.html

[13] M. Hlavová. Approximation of helix in CAD/CAM systems. Proceedings of the 18th Conference on Applied Mathematics (APLIMAT 2019), Bratislava, Slovakia (2019):500 - 507. 